\title{
Effect of serum cystatin C in early diabetic nephropathy in type 2 Iraqi diabetic patients
}

\author{
Ahmed Abed Kadhem Al-Saedy, Kismet M. Turki, Shaymaa Z. Nada ${ }^{a}$
}

¿Department of Biochemistry, College of Medicine, University of Karbala, holy Karbala, Iraq.
'Department of Biochemistry, College of Medicine, University of Baghdad, Baghdad, Iraq.
Correspondence to Ahmed Abed kadhem (email: ahmed_albarkh@yahoo.com).
(Submitted: 17 December 2016 - Revised version received: 28 December 2016 - Accepted: 22 January 2017 - Published online: 26 June 2017)

\begin{abstract}
Objective This study aimed to find the effect of serum Cystatin $C$ in early diabetic nephropathy.
Method This study was conducted in Al Kindy Teaching Hospital during the period from December, 2015 to June, 2016. The study included 90 subjects (30 males and 30 females) with diabetic type 2 and 30 healthy control. Age is between 30 and 70 years. Patients were with no history of liver disease, thyroid or other endocrine diseases through clinical interviewing. They were divided in to three groups. 30 healthy controls, 30 patients with type 2 diabetes mellitus with no albuminuria (albumin excretion in urine $<30 \mu \mathrm{g} / \mathrm{ml}$ ) and 30 patients with type 2 diabetes mellitus with micro albuminuria (albumin excretion in urine $>30 \mu \mathrm{g} / \mathrm{ml}$ ).

Results There were no significant differences in age, body mass index (BMI) among the studied groups $(p>0.05)$. Serum Cystatin C levels showed significant difference $(p<0.001)$ among studied groups, it was significantly higher in micro albuminuria than the other two groups. There was highly significant positive correlation between Cystatin C and serum creatinine $(r=0.697, p<0.001)$, and a significant negative correlation between Cystatin C and GFR $(r=-0.455, p=0.011)$ and showed significant positive correlation between Cystatin $C$ and urine albumin $(r=0.526, p=0.003)$ in type 2 diabetic patients with micro albuminuria.

Conclusion Cystatin C is negatively correlated with the amount of GFR, so that Cystatin C considered reliable and sensitive marker for identifying changes in GFR. In type 2 diabetes mellitus with micro albuminuria serum Cystatin C level considered predict marker for renal failure.

Keywords diabetic nephropathy, micro albuminuria, serum Cystatin C
\end{abstract}

\section{Introduction}

Diabetic nephropathy is a common complication of diabetes mellitus type 2 . The mechanism involved includes changes in blood vessels that supply peripheral nerves and metabolic disorder. ${ }^{1}$ Diabetic nephropathy characterized by an increased excretion of protein, particularly albumin in urine, decline of the glomerular filtration rate (GFR) and elevated blood pressure leading to end stage renal failure. ${ }^{2}$ Diabetic nephropathy has been reported to occur in $25-40 \%$ of people with type 1 or type 2 diabetes. ${ }^{3}$ Early diagnosis of diabetic nephropathy is important, and early therapy decreases the progression of renal disease. ${ }^{4}$

Micro albuminuria was measured in people with diabetes mellitus and people with GFR less than $60\left(\mathrm{ml} / \mathrm{min} / 1.73 \mathrm{~m}^{2}\right)$ and people with strong chronic kidney disease. ${ }^{5}$ Micro albuminuria is used as screening and diagnostic diabetic nephropathy. It is a marker of early diagnosis of kidney disease and also used as a predictor for development coronary heart disease and mortality. ${ }^{6}$ The macro albuminuria increase of albumin and large amount other protein can pass through the urine? Screening for diabetic nephropathy must be initiated at the time of diagnosis in patient with type 2 diabetes. First screening has been recommended at 5 years after diagnosis in patient with poor glycemic and high normal blood pressure levels. ${ }^{8}$ Cystatin C is a $13 \mathrm{kDa}$ protein with low molecular weight and produced by all nucleated cells and freely filtered by the renal glomeruli and reabsorbed in the proximal tubule. Cystatin $\mathrm{C}$ is not affected by age or muscle mass in healthy person. Increased urinary Cystatin $\mathrm{C}$ is a marker of renal tubular dysfunction. ${ }^{9}$ Cystatin $\mathrm{C}$ has multiple biological functions including modulation of the immune system and controlling extracellular proteolysis. Cystatin $\mathrm{C}$ is reabsorbed by proximal tubule epithelial cells and is not returned to the circulation. ${ }^{10}$ Cystatin $\mathrm{C}$ is highly correlated with GFR and not influenced by inflammatory conditions, muscle mass, age, body composition and gender. Increased Cystatin $\mathrm{C}$ is associated with increase cardiovascular morbidity risk and atherosclerosis progression in diabetes and severity obese children. ${ }^{11}$ Cystatin $\mathrm{C}$ is not normally detected in the urine and found in the urine of patients with tubular disease suggesting role as a marker of renal tubular damage. ${ }^{12}$ It is abundant in the serum and less dependent on extra renal factor compared to creatinine and greater sensitivity for revealing mild renal dysfunction even in presumably healthy individuals compared to conventional renal indictor. ${ }^{13}$ Cystatin $C$ levels have been used most commonly to assess kidney function. Serum creatinine and serum Cystatin C are endogenous markers of kidney function. Serum creatinine levels are associated positively with greater muscle mass and dietary meat intake, while, the serum Cystatin $\mathrm{C}$ level is less sensitive to inter-individual differences in muscle mass. The levels of these markers are increased in person with higher body mass index, inflammation, and diabetes. ${ }^{14}$ Many studies show the Cystatin C as it believes as a marker of tubular and glomerular dysfunction for early diabetic nephropathy, and the urine level in Cystatin $\mathrm{C}$ is high in micro albuminuria in diabetic patients compared with normal albuminuria. ${ }^{15}$

This study aimed to test the effect of serum Cystatin $C$ in early diabetic nephropathy in type 2 diabetes mellitus of diabetic Iraqi patients.

\section{Materials and Methods}

This study was conducted in Al Kindy Teaching Hospital during the period from December 2015 to June 2016. The study included 90 subjects (30 males and 30 females) with 
diabetic type 2 and 30 healthy controls. Age is between 30 and 70 years. Patients with no history of liver disease, thyroid or other endocrine disease through clinical interviewing were divided into three groups of 30 healthy controls, 30 type 2 diabetes mellitus with no albuminuria (albumin excretion in urine $<30 \mu \mathrm{g} / \mathrm{ml}$ ) and 30 type 2 diabetes mellitus with micro albuminuria (albumin excretion in urine $>30 \mu \mathrm{g} / \mathrm{ml}$ ). Urine sampling was performed by giving each subject a suitable disposable container with immediate freezing in the deep freeze at $-20^{\circ} \mathrm{C}$. The general physical, chemical and microscopic examinations of urine were performed on another part of urine specimen to check for the final selection or exclusion of a subject in the study. Blood sampling was performed at 8.00-11.00 AM in the fasting state for all the patients and controls, and about $7 \mathrm{ml}$ of venous blood was obtained by antecubital venipuncture using disposable syringes with $\mathrm{G} 21$ needles in the sitting position. Blood was divided into EDTA tube for haemoglobin and plain tube for hemoglobinAlc test $(2.0 \mathrm{ml})$. The blood was allowed to clot for about 20-30 minutes at room temperature, and the serum was recovered by centrifugation at $3000 \mathrm{rpm}$ for 10 minutes and transferred into plain plastic tubes and stored at $-20^{\circ} \mathrm{C}$. All patients' height and weight were measured. Body Mass Index (BMI) was calculated as a ratio of the weight to the height $\left(\mathrm{kg} / \mathrm{m}^{2}\right)$. Blood pressure was measured three times, and the average value was considered for data analysis. Fasting blood glucose (FBG) concentration was determined by using glucose oxidase method. $\mathrm{HbA1c} \%$ assay using the Siemens Dimension clinical chemistry system is an in vitro diagnostic assay for the quantitative determination of $\mathrm{HbAlc}$ in human. Albumin excretion in urine was measured by the fully-auto chemiluminescence immunoassay (CLIA) Analyzer MAGLUMI1000). Serum urea and serum creatinine levels were determined using enzymatic colorimetric methods. GFR was estimated using the modification of diet in renal disease (MDRD) abbreviated equation:

$\left\{\right.$ GFR $=186 \times(\text { serum creatinin })^{-1.154} \times(\text { age })^{-.203} \times(0.742$ if female )\}

and serum Cystatin C was measured using Cosbio kit, enzyme-linked immunosorbent assay (ELISA).

\section{Results}

The result expressed as mean $\pm \mathrm{SD}$ difference between the groups was analyzed by one-way analysis of variance (ANOVA). The correlation between various variables was calculated using the person correlation coefficient. Receiver operating characteristic (ROC) analysis was employed to calculate the area under the curve (AUC) and to find the best cut off values to diagnostic specificity and sensitivity. $p<0.05$ was accepted as statistically significant. All statistical calculations were done using Statistical Package for the Social Science version 20 (SPSS). The result (Table 1) showed no statistically significant difference in terms of age, BMI $(p>0.05)$ between the studied groups. Systolic blood pressure showed a statistically significant difference $(p<0.001)$ among study groups. Diastolic blood pressure also showed statistically significant difference $(p<0.001)$ among study groups. T-test showed statistically significant difference in the duration of diabetes among no albuminuria and micro albuminuria diabetic group. The blood glucose levels showed a statically significant difference $(p<0.001)$ among study groups. It was significantly higher in micro albuminuria than any albuminuria and healthy control. Glycated hemoglobin (HbA1c\%) levels showed a statically significant difference $(p<0.05)$ among studied groups. It was significantly higher in micro albuminuria than any albuminuria and healthy control group. Urine albumin levels showed a significant higher in type 2 diabetic patients with micro albuminuria than type 2 diabetic patients with no albuminuria and healthy control group, which is highly increased significantly $(p<0.001)$. The levels of blood urea were significantly higher in micro albuminuria $(p<0.05)$ when compared with no albuminuria and healthy control group. Serum creatinine levels was significantly higher in micro albuminuria than no albuminuria and healthy Group $(p<0.001)$. The level of glomerular filtration rate (GFR) was significantly higher in micro albuminuria than any albuminuria and healthy group $(p<0.05)$. Serum Cystatin C levels showed significantly increased $(p<0.001)$ among studied groups. It was significantly higher in micro albuminuria than any albuminuria. The correlation analyses revealed the relationship between serum

\begin{tabular}{|c|c|c|c|c|}
\hline Characteristics & Healthy control & No albuminuria & Micro albuminuria & P-ANOVA \\
\hline Age (year) & $53.5 \pm 11.7$ & $53.7 \pm 11.5$ & $55.2 \pm 9.7$ & $>0.05$ \\
\hline BMI $\left(\mathrm{Kg} / \mathrm{m}^{2}\right)$ & $27.5 \pm 4.1$ & $29.21 \pm 4.8$ & $29.4 \pm 5.5$ & $>0.05$ \\
\hline BP (systolic) mm/Hg & $124.2 \pm 12.7$ & $133.0 \pm 17.1$ & $143.0 \pm 16.2$ & $<0.001$ \\
\hline BP (diastolic) mm/Hg & $79.5 \pm 8.1$ & $87.0 \pm 5.8$ & $92.5 \pm 5.0$ & $<0.001$ \\
\hline Duration DM (year) & - & $5.9 \pm 2.0$ & $7.9 \pm 5.6$ & $t$-test $(<0.05)$ \\
\hline $\mathrm{FBS}(\mathrm{mg} / \mathrm{dl})$ & $98.6 \pm 12.4$ & $197.6 \pm 63.0$ & $236 \pm 83.1$ & $<0.001$ \\
\hline $\mathrm{HbA1c}(\%)$ & $4.8 \pm 0.4$ & $7.5 \pm 0.7$ & $8.2 \pm 1.6$ & $<0.001$ \\
\hline Urine albumin ( $\mu \mathrm{g} / \mathrm{ml})$ & $5.3 \pm 1.7$ & $18.7 \pm 4.9$ & $90.9 \pm 69.0$ & $<0.001$ \\
\hline Serum urea (mg/dl) & $34.6 \pm 8.3$ & $32.8 \pm 7.1$ & $43.2 \pm 17.0$ & $<0.001$ \\
\hline Serum creatinine (mg/dl) & $0.7 \pm 18.2$ & $0.8 \pm 21.7$ & $1.1 \pm 26.3$ & $<0.001$ \\
\hline GFR (ml/min/1.73 m²) & $91.6 \pm 18.2$ & $85.6 \pm 21.7$ & $75.1 \pm 26.3$ & $<0.001$ \\
\hline Serum Cystatin C (ng/ml) & $4.6 \pm 3.4$ & $8.3 \pm 4.3$ & $20.4 \pm 16.0$ & $<0.001$ \\
\hline
\end{tabular}

ANOVA $f$-test $p<0.05$ is considered as significant (S); $p<0.001$ is considered as highly significant (HS); and $p>0.05$ considered as non-significant; SD: standard deviation (mean \pm SD); BMI: Body mass index; BP: Blood pressure; DM: Diabetes mellitus; GFR: Glomerular filtration rate. 
Cystatin C and serum creatinine amount GFR, and urine albumin excretion in diabetic patients with micro albuminuria. There was a highly significant positive correlation between Cystatin $\mathrm{C}$ and serum creatinine $(r=0.697, p<0.001)$ as shown in Fig. 1, and significant negative correlation between Cystatin $\mathrm{C}$ and GFR $(r=-0.455, p=0.011)$ as shown Fig. 2 and shows significant Cystatin $\mathrm{C}$ and urine albumin $(r=0.526, p=0.003)$ in type 2 diabetic patients with microalbuminuria as shown in Fig. 3.

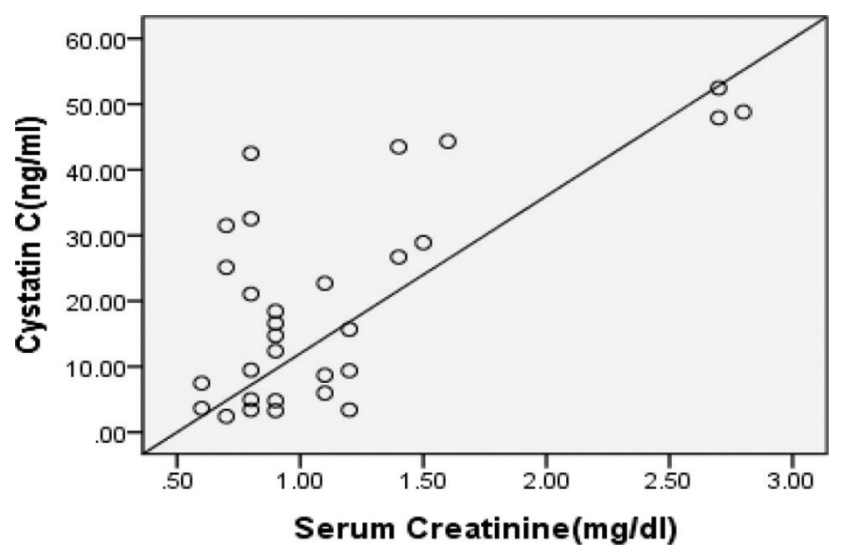

Fig. 1 Relationship between serum Cystatin C and serum creatinine.

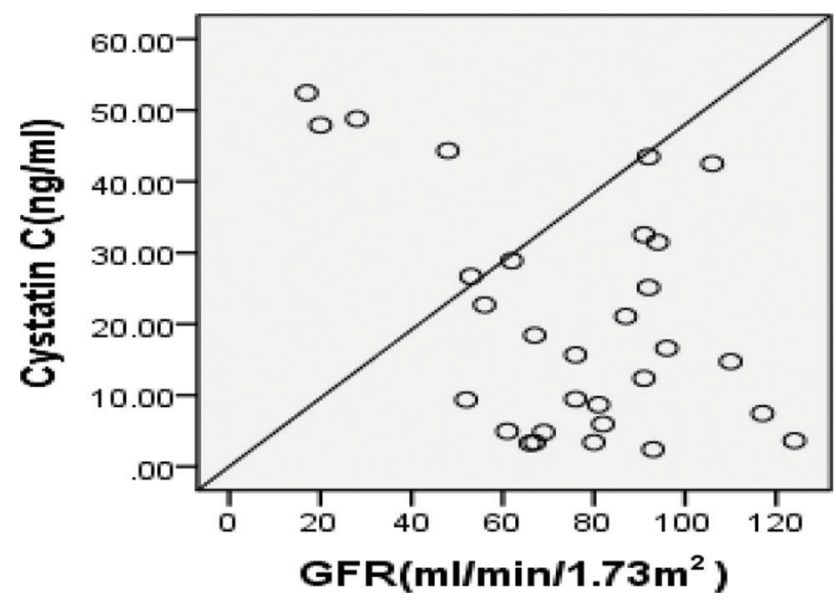

Fig. 2 Relationship between serum Cystatin C and GFR.

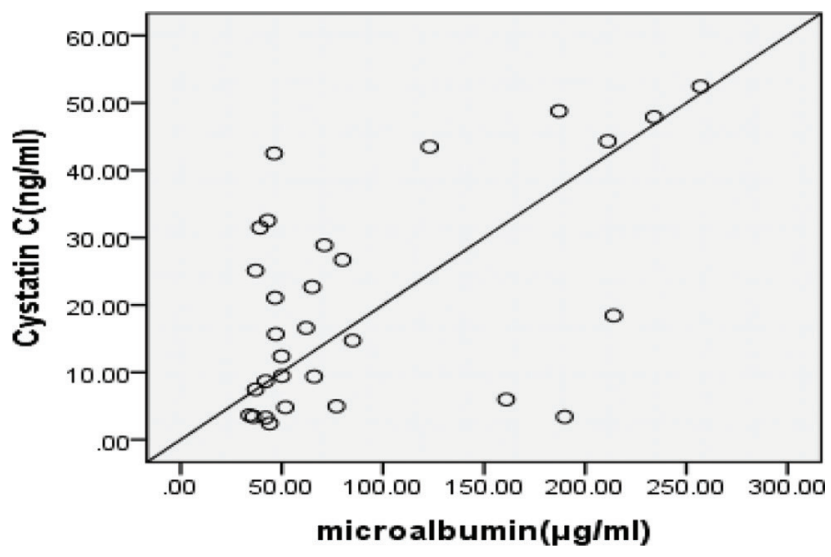

Fig. 3 Relationship between serum Cystatin C and micro albumin $(1, \mathrm{C})$.
ANOVA f-test $p<0.05$ is considered as significant (S). $p<0.001$ is considered as highly significant (HS). And $p>0.05$ considered as non-significant, SD: standard deviation (mean \pm SD), BMI: Body Mass Index, BP: Blood Pressure, DM (Diabetes mellitus), GFR (Glomerular Filtration Rate).

The area under the receiver operator characteristic (ROC) curve was used to discriminate between diabetic patients with micro albuminuria and diabetic patients with no albuminuria depending on levels of analytes of this study. The area under the curve was high for urine albumin $(\mathrm{ROC}=1.0)$, serum urea $(\mathrm{ROC}=0.758)$, serum Cystatin C $(\mathrm{ROC}$ area $=0.706)$, serum creatinine $(\mathrm{ROC}$ area $=0.689)$, fasting blood sugar $(\mathrm{ROC}$ area $=$ $0.638)$, GFR (ROC area $=0.616), \mathrm{HbA1c} \%($ ROC area $=0.586)$, and BMI (ROC area $=0.528)$ as shown in Table 2 and Fig. 4 . The best discriminative cut-off value for each of those analytes was selected, and the sensitivity and specificity of each analytes were calculated to predict diabetic patients with micro albuminuria and to define those with no albuminuria.

Table 2. Area under operator characteristic (ROC) curve for prediction of micro albuminuria in diabetic patients

\begin{tabular}{llc}
\hline Parameter & ROC & P-value \\
\hline Urine albumin $(\mu \mathrm{g} / \mathrm{ml})$ & 1.0 & $<0.0001$ \\
Blood urea $(\mathrm{mg} / \mathrm{dl})$ & 0.758 & $<0.0001$ \\
S. Cystatin C $(\mathrm{ng} / \mathrm{ml})$ & 0.706 & 0.004 \\
Serum creatinine $(\mathrm{mg} / \mathrm{dl})$ & 0.689 & 0.005 \\
FBS $(\mathrm{mg} / \mathrm{dl})$ & 0.638 & 0.06 \\
GFR ml/min/1.73 m² & 0.616 & 0.11 \\
$\mathrm{HbA1} \%$ & 0.586 & 0.25 \\
BMl $\left(\mathrm{Kg} / \mathrm{m}^{2}\right)$ & 0.528 & 0.71 \\
\hline
\end{tabular}

ROC: Reactive Operator Characteristics, $p<0.001$ considered high significant. And $p>0.05$ considered non-significant, FBS (Fasting Blood Sugar), GFR (Glomerular Filtration Rate), HbA1c\% (Hemoglobin A1C), and BMI (Body Mass Index).

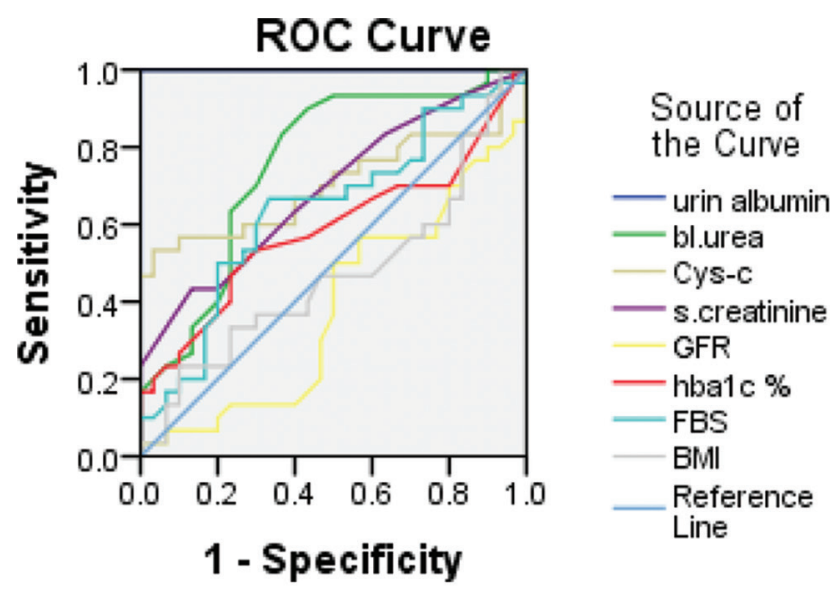

Diagonal segments are produced by ties.

Fig. 4 ROC figure showing the tradeoff between sensitivity (rate true positive result) and 1- specificity (rate of false positive results) for selected measurements when used as a test to predict micro albuminuria in diabetic patients differentiating them from diabetics with normal albuminuria. 
The cut-off value of urine albumin level at $(28.0 \mu \mathrm{g} / \mathrm{ml})$ had a sensitivity $100 \%$ and a specificity $100 \%$. Serum urea level at (31 mg/dl) had a sensitivity of $90 \%$ and a specificity of $56 \%$. Serum Cys-C level at cut-off value of $(15.2 \mathrm{ng} / \mathrm{ml})$ had a sensitivity of $53 \%$ and a specificity $96 \%$. Serum creatinine cut-off level at $(1.0 \mathrm{mg} / \mathrm{dl})$ had a sensitivity $43 \%$ and specificity $86 \%$. Fasting blood sugar level at a cut-off value $(216 \mathrm{mg} / \mathrm{dl}) \mathrm{had}$ a sensitivity $66 \%$ and a specificity $66 \%$. GFR level at a cut-off value (93) had a sensitivity $80 \%$ and a specificity $46 \%$. As shown in Table 3.

ROC : Reactive operator characteristics, $p<0.001$ considered high significant. And $P>0.05$ is considered as nonsignificant, FBS (Fasting blood sugar), GFR (Glomerular Filtration Rate), HbA1c\% (Hemoglobin A1C), and BMI (Body Mass Index)

\section{Discussion}

Various parameters have been recently investigated in serum and urine that play an important role as early detection markers of acute kidney diseases and its complications such as cystatin C, kidney injury molecule- $1 .{ }^{16}$ Diabetic nephropathy is the leading cause end stage renal disease, and hyperglycemia plays a role in the development of diabetic nephropathy. Hyperglycemia causes increase hyper filtration and renal injury. In general, the hyperglycemia is considered as the key initiator for kidney damage by activation of other metabolic pathways and formation or increase in oxidative stress. ${ }^{17}$ The presence of micro albuminuria is considered as indicator or warning signal to renal and cardiovascular disease in patients with type 2 diabetes mellitus, and also considered as the earliest marker of diabetic nephropathy and associated with significant glomerular damage. Studies showed that reduced risk factors lead to decrease level of micro albuminuria and decrease in renal and cardiovascular diseases. ${ }^{18}$ Recent studies showed that the micro albuminuria does not necessary reflect renal impairment in addition to the early structure damage in both tubular structure and glomerular that may be present in normal albuminuria. ${ }^{11}$ So, there is a need to find biomarkers that help in identification of the patients' risk of the disease and monitoring preventive and therapeutic effects. In this study, there is an increased level of Cys-C in diabetic patients with micro albuminuria is highly significant when compared to diabetic patients with no albuminuria and control group. This is in agreement with the results of other study. ${ }^{19}$ Serum Cystatin C performed well as a marker to detect acute renal failure and used to detect development of acute renal failure earlier than serum Creatinine. ${ }^{20}$ Other study reported that serum Cystatin $\mathrm{C}$ is a better early predictor of progression of ESRD in person with type 2

\begin{tabular}{lccc}
\hline Table 3. The cut-off values of some study analytes that best \\
predict micro albuminuria in diabetic patients \\
\hline Parameter & Cut-off & Sensitivity (\%) & Specificity (\%) \\
\hline Urine albumin & 28 & 100 & 100 \\
Blood urea & 31 & 90 & 56 \\
S. Cystatin C & 15.2 & 53 & 96 \\
Serum creatinine & 1.0 & 43 & 86 \\
Fasting blood sugar & 216 & 66 & 66 \\
GFR & 93 & 80 & 46 \\
\hline
\end{tabular}

diabetes, and the ability of serum Cystatin $\mathrm{C}$ to detect pathologies changes in renal function is very high. ${ }^{21}$ Other studies reported that the increase in serum Cystatin $\mathrm{C}$ is more than serum creatinin in kidney damage. Serum Cystatin $\mathrm{C}$ is not influenced by race, muscle mass, gender and age. This may suggest serum Cystatin C equation to calculate GFR that is more important than serum Creatinin equation in calculation of GFR..$^{22}$ The present study shows a statistically significant positive correlation between serum Cys- $\mathrm{C}$ and serum creatinine of diabetic patients with micro albuminuria. This is in agreement with the finding of many studies. ${ }^{23}$ There are negative correlations between serum Cys- $\mathrm{C}$ and the amount of GFR. Serum Cys-C shows significant positive correlation with urine albumin. These results are in agreement with other study who stated that there was a significant positive correlation between serum $\mathrm{Cys}-\mathrm{C}$ and urine albumin exertion. $^{24}$ The micro albuminuria is used as the earliest marker of diabetic nephropathy. However, other study showed that the micro albuminuria is not necessary to reflect renal impairment and suggested that the early structure damage in glomeruli and tubular structure may be present in the normal albuminuria. So, it becomes necessary to find marker to evaluate early tubular damage indecently of albuminuria development and progression in diabetic patients with early nephropathy. ${ }^{11}$

The Cys-C filtered freely by renal glomeruli and reabsorbed in the proximal tubule and has molecular weight of approximately 120 times greater than serum creatinine. However, increased concentration Cystatin $\mathrm{c}$ in the blood indictor effected the kidney or mild renal impairment. ${ }^{23}$ The area under a ROC curve quantifies the ability of the statistics to correctly discriminate between patients into abnormal and normal micro albuminuria. The classification of patients depended on to define urine albumin excretion. Generally, a test in ROC curve with range from 0.5 to 1.0 , however, the area if it was 0.5 , the test did not identify the ability into positive and negative ROC evaluation by reporting $p$ value that tested the null hypotheses. The area under curve really equals 0.5 in others words the $p$ value answers this question. If the $p$ value small that means test dose actually discriminates between abnormal and normal. If the $p$ value is large that means the diagnostic test is not better than flipping a coin to diagnostic patients. The best test to diagnosis disease will be that one with sensitivity and specificity $100 \%$. However, ROC curve is designed for tuning sensitivity and specificity to have comparison between highest sensitivity and highest specificity. ${ }^{17}$ In the study, the urine albumin excretion more active diagnosis test for microalbuminuric patients which goes in line with the patients with higher glucose level and long duration of disease in addition to the serum level Cys-C, serum creatinine, GFR, and serum urea followed with micro albuminuria in diagnostic diabetic nephropathy. This is a useful biomarker to prognosis and detection in patients with micro albuminuria in early stage of diabetic nephropathy. Also in early stage can guide early intervention for the patient's own healthy and prevent disease progression, according to the results of this study all the patients with type 2 diabetes mellitus female and male having high serum Cys-C in early stage of diabetic nephropathy. This analytes showed higher predictive ability to detection and progression of the diseases. 


\section{Conclusion}

Poor glycemic control is characteristic of diabetes patients with micro albuminuria. In the study result show Cys-C level increase with development and progression of diabetic nephropathy. And show Cys-C negative correlated with amount GFR, so that Cys- $\mathrm{C}$ considered a reliable and sensitive marker for identifying changes in GFR. Cys-C used full detection marker of acute renal failure earlier than creatinine .Cystatin $\mathrm{C}$ in diabetic patients with micro albuminuria considered to predict practically well renal events.

\section{Conflict of Interest}

None.

\section{References}

1. Zychowska M, Rojewska E, Przewlocka B, Mika J. Mechanisms and pharmacology of diabetic neuropathy-experimental and clinical studies. Pharmacol Rep. 2013;65:1601-1610.

2. Mise K, Hoshino J, Ueno T, Sumida K, Hiramatsu R, Hasegawa E, et al. Clinical implications of linear immunofluorescent staining for immunoglobulin $\mathrm{G}$ in patients with diabetic nephropathy. Diabet Res Clin Pract. 2014;106: 522-530.

3. Maclsaac RJ, Ekinci El, Jerums G. Markers of and risk factors for the development and progression of diabetic kidney disease. Am J Kidney Dis. 2014;63:S39-S62.

4. Josipović J, Katičić D, Pavlović D. Diabetic nephropathy: diagnosis, prevention and treatment. MEDIX. 19(107/108), 2013.

5. Viberti G, Wheeldon NM. MicroAlbuminuria Reduction with VALsartan (MARVAL) Study Investigators. Microalbuminuria reduction with valsartan in patients with type 2 diabetes mellitus a blood pressure-independent effect. Circulation. 2002;106:672-678.

6. Xia F, Liu G, Shi Y, Zhang Y. Impact of microalbuminuria on incident coronary heart disease, cardiovascular and all-cause mortality: a meta-analysis of prospective studies. Int J Clin Exp Med. 2015;8:1.

7. Jafar TH, Stark PC, Schmid CH, Landa M, Maschio G, de Jong PE, et al. Progression of chronic kidney disease: the role of blood pressure control, proteinuria, and angiotensin-converting enzyme inhibition: a patient-level meta-analysis. Ann Intern Med. 2003;139:244-252.

8. Kim SS, Song SH, Kim IJ, Jeon YK, Kim BH, Kwak IS, et al. Urinary cystatin $\mathrm{C}$ and tubular proteinuria predict progression of diabetic nephropathy. Diabetes Care. 2013;36:656-661.

9. Waheed HJ. A comparative study for Cystatin C and some biochemical markers for predicting diabetic nephropathy in Iraqi patients. Int J Curr Microbiol App Sci. 2015:4:108.

10. Papadopoulou-Marketou N, Skevaki C, Kosteria I, Peppa M, Chrousos GP, Papassotiriou I, et al. NGAL and cystatin C: two possible early markers of diabetic nephropathy in young patients with type 1 diabetes mellitus: one year follow up. Hormones (Athens). 2015;14:232-40.

11. Oh MY, Lee H, Kim JS, Ryu WS, Lee SH, Ko SB, et al. Cystatin C, a novel indicator of renal function, reflects severity of cerebral microbleeds. BMC Neurol. 2014;14:1.

12. Fiseha T. Urinary biomarkers for early diabetic nephropathy in type 2 diabetic patients. Biomar Res. 2015;3: p. 1.

13. Bhavsar NA, Appel LJ, Kusek JW, Contreras G, Bakris G, Coresh J, et al. AASK Study Group, Comparison of measured GFR, serum creatinine, cystatin C, and beta-trace protein to predict ESRD in African Americans with hypertensive CKD. Am J Kidney Dis. 2011;58:886-893.

14. Sun YM, Su Y, Li J, Wang LF. Recent advances in understanding the biochemical and molecular mechanism of diabetic nephropathy. Biochem Biophys Res Commun. 2013;433:359-361.

15. Hawazin Youssef Khalaf. Association between Micro albuminuria and glycosylated hemoglobin, some oxidative stress biomarkers and Atherogenicity in type 2 diabetes women. College of Medicine and committed of postgraduate studies at AL Mustansiriya University. 2015.

16. Al-Tu'ma FJ, Dheyauldeen MH, Al-Saegh RM. Measurement of urinary kidney injury molecule-1 as a predictive biomarker of contrast-induced acute kidney injury. JCMS. 2017;3:178-181.

17. Wang C, Li J, Xue H, Li Y, Huang J, Mai J, et al. Type 2 diabetes mellitus incidence in Chinese: contributions of overweight and obesity. Diabet Res Clin Prac. 2015; 107:424-432.

18. Gray N, Picone G, Sloan F, Yashkin A. Relation between BMI and diabetes mellitus and its complications among US older adults. Southern Med J. 2015;108:29-36.

19. Pavkov ME, Knowler WC, Hanson RL, Williams DE, Lemley KV, Myers BD, et al. Comparison of serum cystatin C, serum creatinine, measured GFR, and estimated GFR to assess the risk of kidney failure in American Indians with diabetic nephropathy. Am J Kidney Dis. 2013;62:33-41.

20. Sit D, Basturk T, Yildirim S, Karagoz F, Bozkurt N, Gunes A. Evaluation of the serum cystatin $C$ values in prediction of indications for hemodialysis in patients with chronic renal failure. Int Urol Nephrol. 2014;46: 57-62.

21. Gompou A, Perrea D, Karatzas T, Bellos JK, Kastania AN, Boletis I, et al. Relationship of changes in Cystatin-C with serum creatinine and estimated glomerular filtration rate in kidney transplantation. Transplant Proc. 2015;47:1662-1674

22. Wang T, Wang Q, Wang Z, Xiao Z, Liu L. Diagnostic value of the combined measurement of serum hcy, serum cys $C$, and urinary microalbumin in type 2 diabetes mellitus with early complicating diabetic nephropathy. ISRN Endocrinol. 2013.

23. Suzuki Y, Matsushita K, Seimiya M, Yoshida T, Sawabe Y, Ogawa M, et al. Serum cystatin C as a marker for early detection of chronic kidney disease and grade 2 nephropathy in Japanese patients with type 2 diabetes. Clin Chem Laborat Med. 2012;50:1833-1839.

24. Sur A. Cystatin C, a better predictor of renal impairment in essential hypertensive patients. Hypertension. 2015;120:80-89.

This work is licensed under a Creative Commons Attribution-NonCommercial 3.0 Unported License which allows users to read, copy, distribute and make derivative works for non-commercial purposes from the material, as long as the author of the original work is cited properly. 